\title{
STABILITY OF SOLUTIONS OF A NONSTANDARD ORDINARY DIFFERENTIAL SYSTEM BY LYAPUNOV'S SECOND METHOD ${ }^{1}$
}

\author{
M. VENKATESULU \\ P.D.N. SRINIVASU \\ Department of Mathematics \\ Sri Sathya Sai Institute of Higher Learning \\ Prasanthinilayam-515 134, Andhra Pradesh, INDIA
}

\begin{abstract}
Differential equations of the form $y^{\prime}=f\left(t, y, y^{\prime}\right)$ where $f$ is not necessarily linear in its arguments represent certain physical phenomena and are known for quite some time. The well known Clairut's and Chrystal's equations fall into this category. Earlier we established the existence of a (unique) solution of the nonstandard initial value problem $y^{\prime}=f\left(t, y, y^{\prime}\right), y\left(t_{0}\right)=y_{0}$ under certain natural hypotheses on $f$. In this paper, we studied the stability of solutions of a nonstandard first order ordinary differential system.
\end{abstract}

Key words: Nonstandard, initial, value, problem, existence, unique, solution, continuous, differentiable, contraction, system, stable, uniform, asymptotic.

AMS (MOS) subject classification: $\quad 34-\mathrm{xx}, 34 \mathrm{Dxx}, 34 \mathrm{D} 20$.

\section{INTRODUCTION}

Differential equations of the form $y^{\prime}=f\left(t, y, y^{\prime}\right)$ where $f$ is not necessarily linear in its arguments represent certain physical phenomena and are known for quite some time. The well known Clairut's and Chrystal's equations fall into this category [4]. A few authors, notably E.L. Ince [5], H.T. Davis [4] et. al. have given some methods for finding solutions of equations of the above type. Apart from these, to the authors knowledge, there does not seem to exist any systematic study of these equations.

In our earlier paper [7], we have studied the solutions of initial value problems (IVPs) associated with the above type of equations. The next fundamental aspect of these equations is the study of stability of solutions. In the present paper, we shall study the stability of solutions of a system of the first order equations of the above type.

Before proceeding to the stability theorems we shall introduce a few notations, definitions and present a few existence results under certain usual assumptions. we shall use the following notation:

\footnotetext{
${ }^{1}$ Received: September, 1990. Revised: December, 1990.
} 
$I=[0, \infty)$

$R^{n}$ denotes the $n$-dimensional real space equipped with the box norm $|\cdot|$ given by

$$
|x|=\sum_{i=1}^{n}\left|x_{i}\right|, \text { where } x=\operatorname{column}\left(x_{1}, x_{2}, \ldots, x_{n}\right) \in R^{n}
$$

$S=\left\{z \in R^{n}|z| \leq c\right\}$, where $c$ is a positive constant,

$D$ is a connected subset of $R^{n}$ with the non empty interior,

$A^{0}$ denotes the interior of a subset $A$ of $R^{n}$,

$C\left(J, R^{n}\right)$ is the set of all continuous vector valued functions defined on a subinterval $J$ of $I$ with values in $R^{n}$,

$y^{\prime}$ and $y^{\prime \prime}$ denote the first and second order derivatives of $y$, respectively, if they exist.

$V_{1} \times V_{2}$ is the Cartesian product of two non-empty sets $V_{1}$ and $V_{2}$, taken in that order,

$f(t, y, z)=\operatorname{column}\left(f_{1}(t, y, z), f_{2}(t, y, z), \ldots, f_{n}(t, y, z)\right)$ is a vector valued not necessarily

linear function defined for $(t, y, z) \in I \times D \times S$ with values in $R^{n}$,

$g_{j}$ denotes $j^{\text {th }}(j=1,2, \ldots, n)$ component of a function $g$ defined on $I \times D \times S$ with values in $R^{n}$

$B^{-1}$ is the inverse matrix of the matrix $B$, if it exists.

Consider the nonstandard initial value problem

$$
\begin{gathered}
y^{\prime}=f\left(t, y, y^{\prime}\right), \\
y\left(t_{0}\right)=y_{0}
\end{gathered}
$$

where $\left(t_{0}, y_{0}\right) \in I \times D^{0}$. Problem (1), (2) will be denoted by NSTD IVP.

Definition 1: By a solution of NSTD IVP (1),(2) we mean a continuously differentiable function $y \in C\left(J, R^{n}\right)$, where $J$ is some subinterval of $I$ containing $t_{0}$, such that

(i) $y\left(t_{0}\right)=y_{0}$,

(ii) $\quad\left(y(t), y^{\prime}(t)\right) \in D \times S$ for all $t \in J$

and

(iii) $\quad y^{\prime}(t)=f\left(t, y(t), y^{\prime}(t)\right)$ holds for all $t \in J$.

We shall denote a solution of problem (1),(2) by $y\left(t, t_{0}, y_{0}\right)$ instead of $y(t)$.

Also, consider the (not necessarily linear) functional equation 


$$
z(\cdot)=f\left(\cdot, y_{0}+\int_{t_{0}} z(s) d s, z(\cdot)\right)
$$

Definition 2: By a solution of equation (3) we mean a function $z \in C\left(J, R^{n}\right)$, where $J$ is some subinterval of $I$ containing $t_{0}$, such that

$$
\left(y_{0}+\int_{t_{0}}^{t} z(s) d s, z(t)\right) \in D \times S \text { for all } t \in J
$$

and
$\left(i i i^{\prime}\right) \quad z(t)=f\left(t, y_{0}+\int_{t_{0}}^{t} z(s) d s, z(t)\right)$ holds for all $t \in J$.

The following result can be easily verified (see [7]):

Result 1: IVP (1), (2) has a [unique] solution if equation (3) has a [unique] solution.

Definition 3: By a solution of system (1) we mean a continuously differentiable function $y \in C\left(J, R^{n}\right)$, where $J$ is some subinterval of $I$, such that conditions (ii) and (iii) of Definition 1 are true for $t \in J$.

Definition f: Let $\phi(t)$ be a solution of system (1) existing on the interval $I$. We say that $\phi(t)$ is a stable solution of system (1) if for every $\epsilon>0$ and $t_{0} \in I$ there exists a $\delta\left(t_{0}, \epsilon\right)>0$ such that whenever $\left|y_{0}-\phi\left(t_{0}\right)\right|<\delta$, where $y_{0} \in D^{0}$, then the solution $y\left(t, t_{0}, y_{0}\right)$ exists for all $t \geq t_{0}$ and $\left|y\left(t, t_{0}, y_{0}\right)-\phi(t)\right|<\epsilon$ for $t \geq t_{0}$.

Let $y(t)$ be an arbitrary solution of (1) existing on the interval $[0,+\infty)$. Substituting the transformation

$$
y=w+\phi
$$

into (1) we get

$$
w^{\prime}=g\left(t, w, w^{\prime}\right)
$$

where $g\left(t, w, w^{\prime}\right)=f\left(t, w+\phi, w^{\prime}+\phi^{\prime}\right)-f\left(t, \phi, \phi^{\prime}\right)$.

We note that $g(t, 0,0)=0$, and that the stability of $\phi(t)$ is equivalent to the stability of the zero solution $(w(t) \equiv 0)$ of system (4). For this reason, from now on we take $D=\left\{y \in R^{n}|y| \leq b\right\}$, where $b>0$ is a constant, little later we assume that $f(t, 0,0)=0$ and we study the stability of the zero solution of (1).

Let $t_{0} \in I$ and $y_{0} \in D^{0}$ (i.e., $\left|y_{0}\right|<b$ ). Assume that the function $f$ satisfies the following 
conditions:

(I) $f(t, y, z)$ is continuous with respect to $(t, y, z) \in I \times D \times S$,

(II) $\quad f(t, y, z) \in S$ for all $(t, y, z) \in I \times D \times S$

and

$$
\begin{aligned}
& \left|f\left(t, y_{1}, z_{1}\right)-f\left(t, y_{2}, z_{2}\right)\right| \leq k_{1}\left|y_{1}-y_{2}\right|+k_{2}\left|z_{1}-z_{2}\right| \text { for all } \\
& \left(t, y_{1}, z_{1}\right),\left(t, y_{2}, z_{2}\right) \in I \times D \times S, \text { where } k_{1} \geq 0 \text { and } 0 \leq k_{2}<1 \text { are constants. }
\end{aligned}
$$

Then we have the following existence result:

Result 2: If conditions $(I)-(I I I)$ are satisfied then IVP (1), (2) has a unique solution existing on the interval $J=\left[t_{0}-\alpha, t_{0}+\alpha\right] \cap I$, where $\alpha$ is any real number such that

$$
0<\alpha<\min \left(\left(1-k_{2}\right) / k_{1},\left(b-\left|y_{0}\right|\right) / c\right) \text {. }
$$

Proof. Consider the Banach space $C\left(J, R^{n}\right)$ equipped with the supremum norm $\|\cdot\|$ given by

$$
\|z\|=\sup _{t \in J}|z(t)|, z \in C\left(J, R^{n}\right)
$$

Let $M$ be the closed subset of $C\left(J, R^{n}\right)$ defined by

$$
M=\left\{z \in C\left(J, R^{n}\right) \mid\|z\| \leq c\right\} .
$$

Denote by $F$ the map on $M$ defined by

$$
(F z)(t)=f\left(t, y_{0}+\int_{t_{0}}^{t} z(s) d s, z(t)\right), z \in M \text { and } t \in J .
$$

Using conditions $(I)-(I I I)$, it is easy to see that $F$ is a contraction on $M$ and by the contraction mapping theorem [6], $F$ has a unique fixed point in $M$. Hence by Result 1, IVP (1), (2) has a unique solution existing on the interval $J$ and the proof is complete.

The following corollary is an immediate consequence of Result 2:

Corollary 1. Let $0<\epsilon<b$ be a constant and let conditions $(I)-(I I I)$ be satisfied. Then for every $y_{0}$ with $\left|y_{0}\right| \leq \epsilon$, IVP (1),(2) has a unique solution existing on the interval $J=\left[t_{0}-\alpha, t_{0}+\alpha\right] \cap I$, where $\alpha$ is any real number, such that

$$
0<\alpha<\min \left(\left(1-k_{2}\right) / k_{1},(b-\epsilon) / c\right) .
$$

In other words, every IVP (1),(2) with $\left|y_{0}\right| \leq \epsilon$ has a unique solution existing on the common 
interval $\mathrm{J}$.

The next result follows from condition $(I I I)$ and the Gronwall's inequality [3], and can be easily established.

Result 3: Let $t_{0} \in I$, and let $y_{0}, \widetilde{y}_{0} \in D^{0}$. Suppose that conditions $(I)-(I I I)$ are true. If the solution $y\left(t, t_{0}, y_{0}\right)$ and $\widetilde{y}\left(t, t_{0}, \widetilde{y}_{0}\right)$ exist on a common interval $J_{1}$ then

$$
\left|y\left(t, t_{0}, y_{0}\right)-\tilde{y}\left(t, t_{0}, \widetilde{y}_{0}\right)\right| \leq\left|y_{0}-\tilde{y}_{0}\right| e^{\frac{k_{1}}{1-k_{2}}\left|t-t_{0}\right|}
$$

for all $t \in J_{1}$.

Now, suppose that $f$ satisfies the following condition:

(IV) $f$ has all continuous partial derivatives upto order $p$ with respect to $(t, y, z) \in I \times D \times S$,

where $p>1$ is an integer, and $\left|\frac{\partial f}{\partial y_{j}}\right| \leq \tilde{k}_{1}, \quad\left|\frac{\partial f}{\partial z_{j}}\right| \leq \tilde{k}_{2}(j=1,2, \ldots, n)$, where $\tilde{k}_{1} \geq 0$ and $0 \leq \tilde{k}_{2}<1$ are constants.

Then, it is easy to verify that condition (IV) implies conditions $(I)$ and $(I I I)$, and hence we have the following result:

Result 4: Suppose that conditions (II) and (IV) are satisfied. Then IVP (1),(2) has a unique solution $y$ existing on the interval $J=\left[t_{0}-\alpha, t_{0}+\alpha\right] \cap I$, where $\alpha$ is given by (6) with $k_{i}=\widetilde{k}_{i}(i=1,2)$. Moreover, $y$ is $p+1$ times continuously differentiable on $J$ and

$$
y^{\prime \prime}=\left(I-\left(\frac{\partial f_{i}}{\partial z_{j}}\right)\right)^{-1}\left(\frac{\partial f}{\partial t}+\left(\frac{\partial f_{i}}{\partial y_{j}}\right) f\right)
$$

where $I$ is $n \times n$ identity matrix

$$
\left(\frac{\partial f_{i}}{\partial z_{j}}\right)=\left[\begin{array}{ccc}
\frac{\partial f_{1}}{\partial z_{1}} & \cdots & \frac{\partial f_{1}}{\partial z_{n}} \\
\cdot & \cdot \\
\cdot & & \cdot \\
\cdot & \cdot \\
\frac{\partial f_{n}}{\partial z_{1}} & \cdots & \frac{\partial f_{n}}{\partial z_{n}}
\end{array}\right]
$$


and $\left(\frac{\partial f_{i}}{\partial y_{j}}\right)$ is defined similarly. Relation (9) follows from the quotient

$$
\frac{y^{\prime}(t+h)-y^{\prime}(t)}{h}=\frac{f\left(t+h, y(t+h), y^{\prime}(t+h)\right)-f\left(t, y(t), y^{\prime}(t)\right)}{h}
$$

by applying the mean value theorem for vector valued functions on the right hand side function, by making use of condition (IV) and, finally, by allowing $h$ tend to zero.

\section{STABILITY THEOREMS}

Throughout this section, we assume that the function $f$ satisfies conditions $(I I)$ and $(I V)$ and that $f(t, 0,0)=0$ for all $t \in I$.

Consider the system

$$
\left.y^{\prime}=f t, y, y^{\prime}\right)
$$

Definition 5: We say that $y(t) \equiv 0$ is a stable solution of (10) if for every $\epsilon>0$ and any $t_{0} \in I$ there exists a $\delta\left(\epsilon, t_{0}\right)>0$ such that whenever $\left|y_{0}\right|<\delta$ then the solution $y\left(t, t_{0}, y_{0}\right)$ exists for all $t \geq t_{0}$ and $\left|y\left(t, t_{0}, y_{0}\right)\right|<\epsilon$ for all $t \geq t_{0}$.

Definition 6: We say that $y(t) \equiv 0$ is a uniformly stable solution of (10) if $\delta$ in Definition 5 is independent of $t_{0}$.

Definition 7: We say that $y(t) \equiv 0$ is an asymptotically stable solution of (10) if

$$
y(t) \equiv 0 \text { is stable }
$$

and

(ii) there exists a $\delta_{0}\left(t_{0}\right)>0$ such that whenever $\left|y_{0}\right|<\delta_{0}$ then the solution $y\left(t, t_{0}, y_{0}\right)$ tends to zero as $t \rightarrow \infty$.

Definition 8: We say that $y(t) \equiv 0$ is an uniformly asymptotically stable solution of $(10)$ if $y(t) \equiv 0$ is uniformly stable

and

(ii) there exists a $\delta_{0}>0$ and for every $\epsilon>0$ there exists a $T(\epsilon)>0$ such that whenever $\left|y_{0}\right|<\delta_{0}$ then $\left|y\left(t, t_{0}, y_{0}\right)\right|<\epsilon$ for all $t \geq t_{0}+T(\epsilon)$.

Note 1: We note that uniform [asymptotic] stability always implies [asymptotic] stability of the zero solution of (10). Below, we shall see that the converse is also true if $f$ is either periodic in $t$ or autonomous. 
Theorem 1: Let $f(t, y, z)$ be periodic in $t$ of period $w$. If the zero solution of (10) is [asymptotically] stable then it is uniformly [asymptotically] stable.

The theorem can be proved easily, using Result 3, along the same lines as of Theorems 7.3 and 7.4. [8], and hence the proof will be omitted here.

Definition 9: We call a real valued function $V(t, y, z)$, defined on $I \times D \times S$, a Lyapunov function, if:

$$
V(t, y, z) \text { is continuous with respect to }(t, y, z) \in I \times D \times S
$$

and

(ii) $V(t, y, z)$ is continuously differentiable with respect to $(t, y, z) \in I \times D^{0} \times S^{0}$.

Definition 10: We define the derivative of $V(t, y, z)$ with respect to system (10) by

$$
V^{*}(t, y, z)=\frac{\partial V}{\partial t}+\sum_{j=1}^{n}\left(\frac{\partial V}{\partial y_{j}}\right) f_{j}+\sum_{j-1}^{n} \frac{\partial V}{\partial z_{j}}\left(\left(I-\left(\frac{\partial f_{i}}{\partial z_{j}}\right)\right)^{-1}\left(\frac{\partial f}{\partial t}+\left(\frac{\partial f_{i}}{\partial y_{j}}\right) f\right)\right)_{j}
$$

Note 2: Along a solution $y$ of system (10), we have, by (9),

$$
\frac{d V}{d t}\left(t, y(t), y^{\prime}(t)\right)=V^{*}\left(t, y(t), y^{\prime}(t)\right)
$$

Definition 11: A real valued function $a(r)$ defined on the interval $[0, b]$ is called positive definite if $a(0)=0$ and $a(r)>0$ for $r \in(0, b]$.

Now, we shall present few theorems for [uniform] stability and [uniform] asymptotic stability of the zero solution of system (10).

Theorem 2 (Stability of the zero solution): Suppose that there exists a Lyapunov function $V(t, y, z)$ defined on $I \times D \times S$ satisfying the following conditions:

(i) $\quad V(t, 0,0)=0$ for all $t \in I$,

(ii) there exists a continuous positive definite function $a(r)$ defined on $[0, b]$ satisfying the inequality a $(|y|) \leq V(t, y, z)$ for all $(t, y, z) \in I \times D \times S$ such that $z=f(t, y, z)$

and

(iii) $\quad V^{*}(t, y, z) \leq 0$ for all $(t, y, z) \in I \times D^{0} \times S^{0}$ such that $z=f(t, y, z)$.

Then the zero solution of system (10) is stable.

We note that for every $(t, y) \in I \times D$, in view of conditions $(I I)$ and $(I V)$, there exists a unique $z \in S$ such that $z=f(t, y, z)$. 
Proof: Let $\epsilon$ be such that $0<\epsilon<b$ and $\left(\tilde{k}_{1} /\left(1-\tilde{k}_{2}\right)\right) \epsilon<c$. From condition (i) and from the continuity of $V$, it follows that for every $t_{0} \in I$ there exists a number $\delta_{0}\left(t_{0}, \epsilon\right)>0$ such that if $\left|y_{0}\right|<\delta_{0}(<\epsilon)$ and $\left|z_{0}\right|<\left(\tilde{k}_{1} /\left(1-\tilde{k}_{2}\right)\right) \delta_{0}$, then

$$
V\left(t_{0}, y_{0}, z_{0}\right)<a(\epsilon)
$$

By Corollary 1 , the solution $y\left(t, t_{0}, y_{0}\right)$ exists initially on the interval $\left[t_{0}, t_{0}+\alpha\right]$, where $\alpha$ is given by (7). We have, by condition (10), that

$$
\left|y^{\prime}\left(t_{0}\right)\right| \leq\left(\frac{\widetilde{k}_{1}}{1-\widetilde{k}_{2}}\right)\left|y_{0}\right|<\left(\frac{\widetilde{k}_{1}}{1-\widetilde{k}_{2}}\right) \delta_{0}
$$

Hence, from relation (12), we get that

$$
V\left(t_{0}, y\left(t_{0}\right), y^{\prime}\left(t_{0}\right)\right)<a(\epsilon)
$$

Claim: $\left|y\left(t, t_{0}, y_{0}\right)\right|<\epsilon$ for all $t \in\left[t_{0}, t_{0}+\alpha\right]$.

For, if possible, let there exist a $t_{1} \in\left(t_{0}, t_{0}+\alpha\right]$ such that $\left|y\left(t, t_{0}, y_{0}\right)\right|=\epsilon$. From condition (iii) and note 2 , we get that

$$
\frac{d V}{d t}\left(t, y\left(t, t_{0}, y_{0}\right), y^{\prime}\left(t, t_{0}, y_{0}\right)\right) \leq 0 \text { for all } t \in\left[t_{0}, t_{1}\right]
$$

which upon integrating between $t_{0}$ and $t$, gives that

$$
V\left(t, y\left(t, t_{0}, y_{0}\right), y^{\prime}\left(t, t_{0}, y_{0}\right)\right) \leq V\left(t_{0}, y\left(t_{0}\right), y^{\prime}\left(t_{0}\right)\right)<a(\epsilon)
$$

for all $t \in\left[t_{0}, t_{1}\right]$. Then from condition (ii) and inequality (13), we get that

$$
\begin{gathered}
a(\epsilon) \leq V\left(t_{1}, y\left(t_{1}, t_{0}, y_{0}\right), y^{\prime}\left(t_{1}, t_{0}, y_{0}\right)\right) \\
\leq V\left(t_{0}, y_{0}, y^{\prime}\left(t_{0}\right)\right)<a(\epsilon),
\end{gathered}
$$

which is a contradiction. Hence our claim is true.

Next, let us denote the solution $y\left(t, t_{0}, y_{0}\right)$ by $y_{1}(t)$. Since $\left|y_{1}\left(t_{0}+\alpha\right)\right|<\epsilon$, again by Corollary 1 , the solution $y\left(t, t_{0}+\alpha, y_{1}\left(t_{0}+\alpha\right)\right)$ of the following initial value problem

$$
y^{\prime}=f\left(t, y, y^{\prime}\right), y\left(t_{0}+\alpha\right)=y_{1}\left(t_{0}+\alpha\right)
$$

exists on the interval $\left[t_{0}, t_{0}+2 \alpha\right]$. If we denote $y\left(t, t_{0}+\alpha, y_{1}\left(t_{0}+\alpha\right)\right)$ by $y_{2}(t)$, we have, by relation (8), that

$$
y_{2}(t)=y_{1}(t) \text { for all } t \in\left[t_{0}, t_{0}+\alpha\right]
$$


and it can be shown as in the above that

$$
\left|y_{2}(t)\right|<\epsilon \text { for all } t \in\left[t_{0}, t_{0}+2 \alpha\right]
$$

Again, since $\left|y_{2}\left(t_{0}+2 \alpha\right)\right|<\epsilon$, by Corollary 1 , the solution $y_{3}(t)$ of the initial value problem

$$
y^{\prime}=f\left(t, y, y^{\prime}\right), y\left(t_{0}+2 \alpha\right)=y_{2}\left(t_{0}+2 \alpha\right)
$$

exists on the interval $\left[t_{0}+\alpha, t_{0}+3 \alpha\right]$, and we have that

and

$$
y_{3}(t)=y_{2}(t) \text { for } t \in\left[t_{0}+\alpha, t_{0}+2 \alpha\right]
$$

$$
\left|y_{3}(t)\right|<\epsilon \text { for all } t \in\left[t_{0}+\alpha, t_{0}+3 \alpha\right] \text {. }
$$

Proceeding in this way, indefinitely, we get a sequence of solutions $\left\{y_{n}(t)\right\}$ and if we define

$$
y(t)=\mid \begin{aligned}
& y_{1}(t), t \in\left[t_{0}, t_{0}+\alpha\right], \\
& y_{2}(t), t \in\left[t_{0}+\alpha, t_{0}+2 \alpha\right] \\
& \cdot \\
& \cdot \\
& y_{n}(t), t \in\left[t_{0}+(n-1) \alpha, t_{0}+n \alpha\right] \\
& \cdot
\end{aligned}
$$

then clearly $y(t)$ is a unique solution of system (10) with $y\left(t_{0}\right)=y_{0}$. Moreover, $y(t)$ exists on the entire interval $\left[t_{0},+\infty\right)$ and $|y(t)|<\epsilon$ for all $t \geq t_{0}$. This shows that the zero solution of (10) is stable and the proof is complete.

Theorem 3 (Uniform stability of the zero solution): Let condition (ii) in Theorem 2 be replaced by the condition:

(ii') $\quad a(|y|) \leq V(t, y, z) \leq b(|y|)$ for all $(t, y, z) \in I \times D \times S$ such that $z=f(t, y, z)$, where $a(r)$ and $b(r)$ are continuous, positive definite functions defined on $[0, b]$.

Then the zero solution of system (10) is uniformly stable.

Proof: Let $\epsilon$ be such that $0<\epsilon<b$ and $\left(\tilde{k}_{1} /\left(1-\widetilde{k}_{2}\right)\right) \epsilon<c$. Choose a $\delta(\epsilon)>0$ such that $b(\delta)<a(\epsilon)$ and $\delta<\epsilon$. Now, let $t_{0} \in I$ and $\left|y_{0}\right|<\delta$. By Corollary 1 , the solution $y\left(t, t_{0}, y_{0}\right)$ exists initially on the interval $\left[t_{0}, t_{0}+\alpha\right]$, where $\alpha$ is given by (7). 
Claim: $\left|y\left(t, t_{0}, y_{0}\right)\right|<\epsilon$ for all $t \in\left[t_{0}, t_{0}+\alpha\right]$.

For, if possible, let there exist a point $t_{2} \in\left(t_{0}, t_{0}+\alpha\right]$ such that $\left|y\left(t_{2}, t_{0}, y_{0}\right)\right|=\epsilon$. Since $\left|y_{0}\right|<\delta<\epsilon$, there exists a point $t_{1} \in\left(t_{0}, t_{2}\right)$ such that $y\left(t_{1}, t_{0}, y_{0}\right)=\delta$. Now, from condition (iii) and note 2 , we get

$$
\frac{d V}{d t}\left(t, y\left(t, t_{0}, y_{0}\right), y^{\prime}\left(t, t_{0}, y_{0}\right)\right) \leq 0 \text { for all } t \in\left[t_{0}, t_{2}\right]
$$

which upon integrating between $t_{1}$ and $t_{2}$, gives that

$$
V\left(t_{2}, y\left(t_{2}, t_{0}, y_{0}\right), y^{\prime}\left(t_{2}, t_{0}, y_{0}\right)\right) \leq V\left(t_{1}, y\left(t_{1}, t_{0}, y_{0}\right), y^{\prime}\left(t_{1}, t_{0}, y_{0}\right)\right) \text {. }
$$

Then from condition $\left(i i^{\prime}\right)$ and inequality (14), we get that

$$
\begin{gathered}
b(\delta)<a(\epsilon) \leq V\left(t_{2}, y\left(t_{2}, t_{0}, y_{0}\right), y^{\prime}\left(t_{2}, t_{0}, y_{0}\right)\right) \\
\leq V\left(t_{1}, y\left(t_{1}, t_{0}, y_{0}\right), y^{\prime}\left(t_{1}, t_{0}, y_{0}\right)\right) \leq b(\delta),
\end{gathered}
$$

which is a contradiction. Hence our claim is true.

Rest of the proof follows as in Theorem 2 and hence will be omitted.

Theorem 4 (Asymptotic stability of the zero solution): Suppose that there exists a Lyapunov function $V(t, y, z)$ defined on $I \times D \times S$ satisfying the following properties:

$$
V(t, 0,0)=0 \text { for all } t \in I \text {, }
$$

$$
a(|y|) \leq V(t, y, z) \text { for all }(t, y, z) \in I \times D \times S \text { such that } z=f(t, y, z) \text {, where } a(r) \text { is a }
$$
continuous, positive definite function defined on $[0, b]$

and

(iii) $\quad V^{*}(t, y, z) \leq-c(|y|)$ for all $(t, y, z) \in I \times D^{0} \times S^{0}$ such that $z=f(t, y, z)$, where $c(r)$ is a continuous, positive definite function defined on $[0, b]$.

Then the zero solution of $(10)$ is asymptotically stable.

Stability of the zero solution of (10) follows from Theorem 2, and proof of existence of a $\delta_{0}\left(t_{0}\right)>0$ such that $\left|y_{0}\right|<\delta_{0}$ implies that the solution $y\left(t, t_{0}, y_{0}\right) \rightarrow 0$ as $t \rightarrow \infty$ can be given along the same lines as that of Theorem $8.5[8]$ and hence will be omitted.

Theorem 5 (Uniform asymptotic stability of the zero solution): Suppose that there exists a Lyapunov function $V(t, y, z)$ defined on $I \times D \times S$ satisfying the following conditions:

(i) $\quad a(|y|) \leq V(t, y, z) \leq b(|y|)$ for all $(t, y, z) \in I \times D \times S$ such that $z=f(t, y, z)$, where $a(r)$ and $b(r)$ are continuous, positive definite functions defined on $[0, b]$

and

$$
V^{*}(t, y, z) \leq-c(|y|) \text { for all }(t, y, z) \in I \times D^{0} \times S^{0} \text { such that } z=f(t, y, z) \text {, where } c(r) \text { is a }
$$


continuous, positive definite function defined on $[0, b]$.

Then the zero solution of (10) is uniformly asymptotically stable.

Proof: By Theorem 3, the zero solution of (10) is uniformly stable. Choose $\eta>0$ such that $\eta<b$ and $\left(\widetilde{k}_{1} /\left(1-\widetilde{k}_{2}\right)\right) \eta<c$. Then there exists a $\delta_{0}>0$ such that if $t_{0} \in I$ and $\left|y_{0}\right|<\delta_{0}$, then $\left|y\left(t, t_{0}, y_{0}\right)\right|<\eta$ for all $t \geq t_{0}$. Also for a given $\epsilon>0$ with $\epsilon<\eta$, there exists a $\delta(\epsilon)>0$ such that if $t_{0} \in I$ and $\left|y_{0}\right|<\delta$, then $\left|y\left(t, t_{0}, y_{0}\right)\right|<\epsilon$ for all $t \geq t_{0}$.

Let

$$
\begin{aligned}
& M_{1}=\inf \{a(r) \mid \delta / 2 \leq r \leq b\}, \\
& M_{2}>\sup \{b(r) \mid \delta / 2 \leq r \leq b\},
\end{aligned}
$$

and

$$
M_{3}=\inf \{c(r) \mid \delta / 2 \leq r \leq b\} .
$$

Clearly $M_{i}>0,(i=1,2,3)$ and, from condition (ii), we have that $M_{1}<M_{2}$. Now, let $T=\left(M_{2}-M_{1}\right) / M_{3}$ and note that $T>0$ and depends only on $\epsilon$.

Claim: For every solution $y\left(t, t_{0}, y_{0}\right)$ of $(10)$ such that $t_{0} \in I$ and $\left|y_{0}\right|<\delta_{0}$, there exists at least one point $t_{1} \in\left[t_{0}, t_{0}+T\right]$ such that $\left|y\left(t_{1}, t_{0}, y_{0}\right)\right|<\delta$.

For, if possible, let there exist a solution $y\left(t, t_{0}, y_{0}\right)$ of $(10)$, for some $t_{0} \in I$ and some $y_{0}$ with $\left|y_{0}\right|<\delta_{0}$, such that $\left|y\left(t, t_{0}, y_{0}\right)\right| \geq \delta$ for all $t \in\left[t_{0}, t_{0}+T\right]$. Then, there exists a $\tilde{t}>t_{0}+T$ such that $\left|y\left(t, t_{0}, y_{0}\right)\right|>\delta / 2$ for all $t \in\left[t_{0}, \tilde{t}\right)$. Now, from conditions (i) and (ii) and note 2, we get that

$$
\begin{gathered}
M_{1} \leq a\left(\left|y\left(t, t_{0}, y_{0}\right)\right|\right) \leq V\left(t, y\left(t, t_{0}, y_{0}\right), y^{\prime}\left(t, t_{0}, y_{0}\right)\right) \\
\leq V\left(t_{0}, y_{0}, y^{\prime}\left(t_{0}\right)\right)-M_{3}\left(t-t_{0}\right) \\
\leq b\left(\left|y_{0}\right|\right)-M_{3}\left(t-t_{0}\right) \\
\leq M_{2}-M_{3}\left(t-t_{0}\right)
\end{gathered}
$$

which implies that

$$
t-t_{0} \leq\left(M_{2}-M_{1}\right) / M_{3}=T \text { for all } t \in\left[t_{0}, \tilde{t}\right),
$$

which is certainly a contradiction. Hence our claim is true.

Thus, we have that if $t_{0} \in I$ and $\left|y_{0}\right|<\delta_{0}$, then $\left|y\left(t_{1}, t_{0}, y_{0}\right)\right|<\delta$ for some $t_{1} \in\left[t_{0}, t_{0}+T\right]$ and consequently $\left|y\left(t, t_{0}, y_{0}\right)\right|<\epsilon$ for all $t \geq t_{0}+T$. The proof is complete. 
The following corollary follows immediately from Theorem 5.

Corollary 2: Let condition (ii) in Theorem 5 be replaced by $\left(i i^{\prime}\right) \quad V^{*}(t, y, z) \leq-c V(t, y, z)$ for all $(t, y, z) \in I \times D^{0} \times S^{0}$ such that $z=f(t, y, z)$, where $c>0$ is a constant.

Then the zero solution of (10) is uniformly asymptotically stable.

\section{EXAMPLES}

1. In the study of free oscillations of positively damped systems, we encounter the following differential equation [1]:

$$
u^{\prime \prime}+w^{2} u+\epsilon\left(u^{\prime}\right)^{3}\left(u^{\prime \prime}\right)^{2}=0
$$

where $w$ is a nonzero constant and $0<\epsilon<<1$.

Equation (15) is equivalent to the system

$$
y^{\prime}=f\left(t, y, y^{\prime}\right)
$$

where

$$
y=\left(\begin{array}{l}
y_{1} \\
y_{2}
\end{array}\right), f=\left(\begin{array}{l}
f_{1} \\
f_{2}
\end{array}\right)=\left(\begin{array}{c}
y_{2} \\
-\left(w^{2} y_{1}+\epsilon z_{1}^{3} z_{2}^{2}\right)
\end{array}\right)
$$

and

$$
y_{1}=u
$$

Choose the constants $b>0$ and $c>0$ such that

$$
3 \epsilon<1 / c^{4} \text { and } b<c\left(1-\epsilon c^{4}\right) /\left(1+w^{2}\right) .
$$

Let $D=\left\{y \in R^{2}|| y \mid \leq b\right\}$ and $S=\left\{z \in R^{2}|| z \mid \leq c\right\}$. We can easily verify that $f(t, y, z)$ is defined on $I \times D \times S$ and satisfies the following conditions:

A) $\quad f(t, 0,0)=0$,

B) $f(t, y, z) \in S$ for $(t, y, z) \in I \times D \times S$,

C) $f(t, y, z)$ is continuously differentiable with respect to $(t, y, z) \in I \times D \times S$, and

$$
\left|\frac{\partial f}{\partial y_{1}}\right| \leq w^{2},\left|\frac{\partial f}{\partial y_{2}}\right| \leq 1,\left|\frac{\partial f}{\partial z_{1}}\right| \leq 3 \epsilon c^{4} \text { and }\left|\frac{\partial f}{\partial z_{2}}\right| \leq 2 \epsilon c^{4} \text {. }
$$


Thus $f$ satisfies conditions $(I I)$ and $(I V)$ on $I \times D \times S$ with $\tilde{k}_{1}=\max \left(1, w^{2}\right)$ and $\tilde{k}_{2}=3 \epsilon c^{4}$.

Now, consider the function $V(t, y, z)$ defined on $I \times D \times S$ by $V(t, y, z)=w^{2} y^{2}{ }_{1}+y_{2}^{2}$. Clearly, $V$ is continuously differentiable on $I \times D \times S$ and satisfies the following conditions:

$$
V(t, 0,0)=0
$$

(ii) $\quad a(|y|) \leq V(t, y, z) \leq b(|y|)$ for all $(t, y, z) \in I \times D \times S$, where $a(r)=\alpha r^{2}, b(r)=\alpha r^{2}$, $\alpha=\min \left(1, w^{2}\right), \beta=\max \left(1, w^{2}\right)$

and

(iii)

$$
\begin{aligned}
V^{*}(t, y, z)=\frac{\partial V}{\partial y_{1}} f_{1}+\frac{\partial V}{\partial y_{2}} f_{2} & \\
= & 2 y_{1} y_{2} w^{2}-2 y_{2}\left(w^{2} y_{1}+\epsilon z_{1}^{3} z_{2}^{2}\right) \\
& =-2 \epsilon y_{2} z_{1}^{3} z_{2}^{2}=-2 \epsilon z_{1}^{4} z_{2}^{2} \leq 0
\end{aligned}
$$

for $(t, y, z) \in I \times D \times S$ such that $z=f(t, y, z)$.

Hence, by Theorem 3, the zero solution of (16) and hence of (15) is uniformly stable.

2. Let

$$
g(y)=\mid \begin{array}{cc}
\sin y, & -\pi / 2 \leq y \leq \pi / 2 \\
1, & \pi / 2<y \\
-1, & y<-\pi / 2 .
\end{array}
$$

Clearly, $g$ is a continuously differentiable function on $R, g(0)=0, y g(y)>0$ for $y \neq 0$ and $|g(y)| \leq 1$

Consider the equation

$$
y^{\prime}=-\alpha g(y)\left(\cos y^{\prime}\right)^{2}
$$

where $0<\alpha \leq 1 / 2$. Let

$$
D=\{y \in R|| y \mid \leq \pi\} \text { and } S=\{z \in R|| z \mid \leq 1\} .
$$

It is easy to verify that the function $f(t, y, z)=-\alpha g(y)(\cos z)^{2}$ satisfies conditions $(I I)$ and $(I V)$ on $I \times D \times S$ and $f(t, 0,0)=0$ for all $t \in I$. Now, consider $V(t, y, z)$ defined on $I \times D \times S$ by $V(t, y, z)=y^{2}$. Clearly, $V$ is continuously differentiable on $I \times D \times S$ and satisfies the following conditions: 


$$
a(|y|) \leq V(t, y, z) \leq b(|y|) \text { for all }(t, y, z) \in I \times D \times S, \text { where } a(r)=b(r)=r^{2},
$$

$$
V(t, 0,0)=0 \text { for all } t \in I
$$

and

(iii) $\quad v^{*}(t, y, z)=\frac{\partial V}{\partial y} f=-2 \alpha y g(y)(\cos z)^{2} \leq-c(|y|)$, for all $(t, y, z) \in I \times D \times S$, where $c(r)=2 \alpha r g(r)(\cos 1)^{2}$.

Hence, by Theorem 5, the zero solution of (18) is uniformly asymptotically stable.

\section{ACKNOWLEDGEMENT}

The authors are extremely grateful to the referee for the valuable and important suggestions which resulted in the present form of the paper. The authors dedicate the paper to the Chancellor of the Institute Bhagawan Sri Sathya Sai Baba.

\section{REFERENCES}

[1] Ali Hasan Nayfeh, "Problems in Perturbations", Wiley, New York, (1985).

[2] Bartle, R.G., “The Elements of Real Analysis", Wiley, New York, (1976).

[3] Brauer, F. and Nohel, J.A., “The Qualitative Theory of Ordinary Differential Equations An Introduction", W.A. Benjamin, New York, (1969).

[4] Davis, H.T., "Introduction to Nonlinear Differential and Integral Equations", Dover Publications, New York, (1962).

[5] Ince, E.L., “Ordinary Differential Equations", Dover Publications, New York, (1956).

[6] Krasnosel'skii, M.A., et. al., “Approximate Solution of Operator Equations”, WoltersNoordhoff Publishing, Groningen, (1972).

[7] Venkatesulu, M. and Srinivasu, P.D.N., "Solutions of nonstandard initial value problems for a first order ordinary differential equation", J. Appl. Math. Sim., Vol 2, No. 4, (1989), 225-237.

[8] Yoshizawa, T., "Stability Theory by Lyapunov's Second Method", The Mathematical Society of Japan, Tokyo, (1966). 


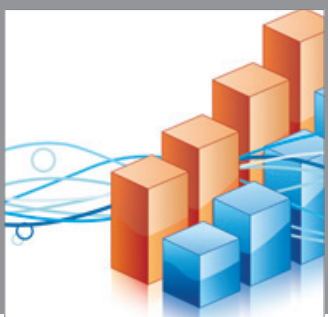

Advances in

Operations Research

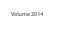

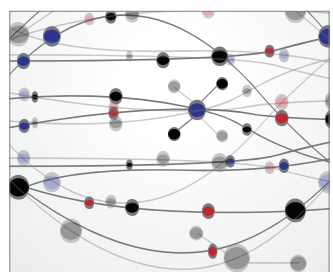

\section{The Scientific} World Journal
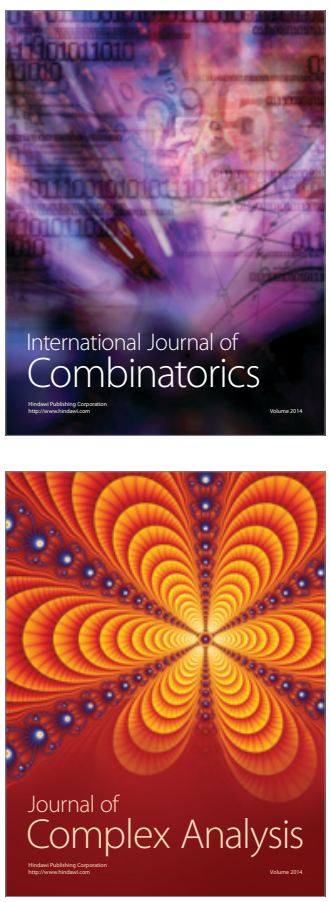

International Journal of

Mathematics and

Mathematical

Sciences
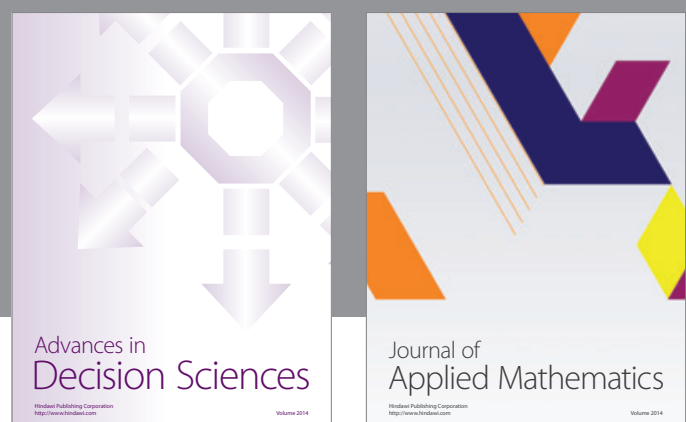

Journal of

Applied Mathematics
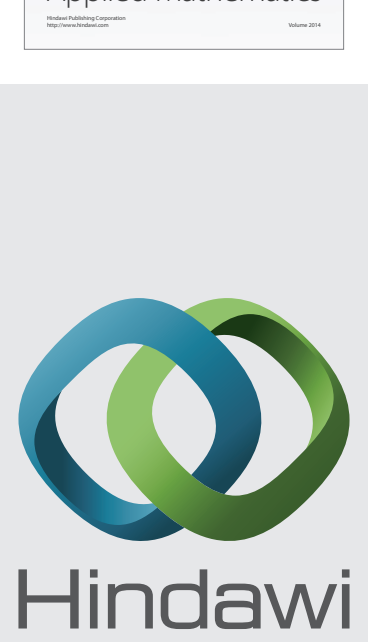

Submit your manuscripts at http://www.hindawi.com
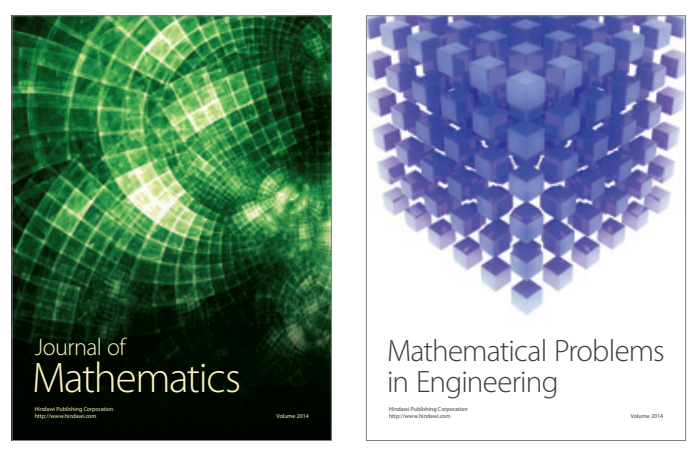

Mathematical Problems in Engineering
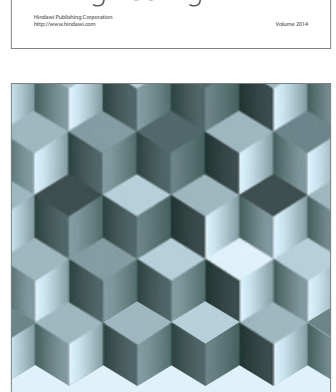

Journal of

Function Spaces
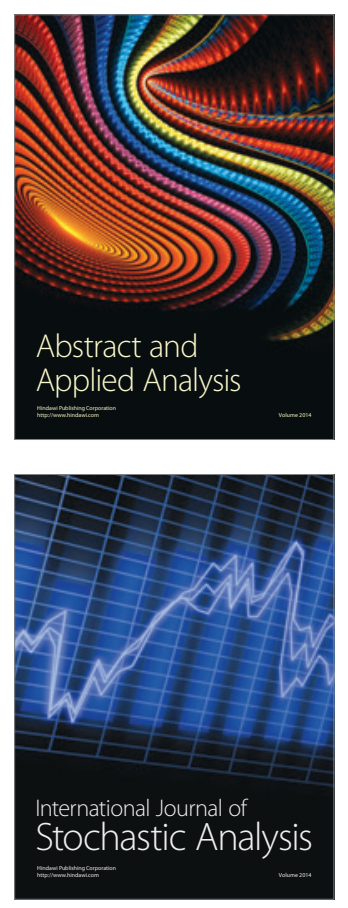

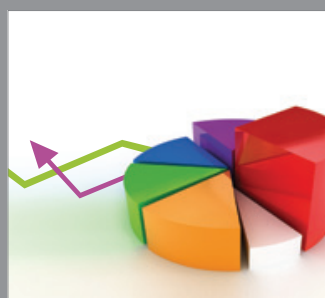

ournal of

Probability and Statistics

Promensencen
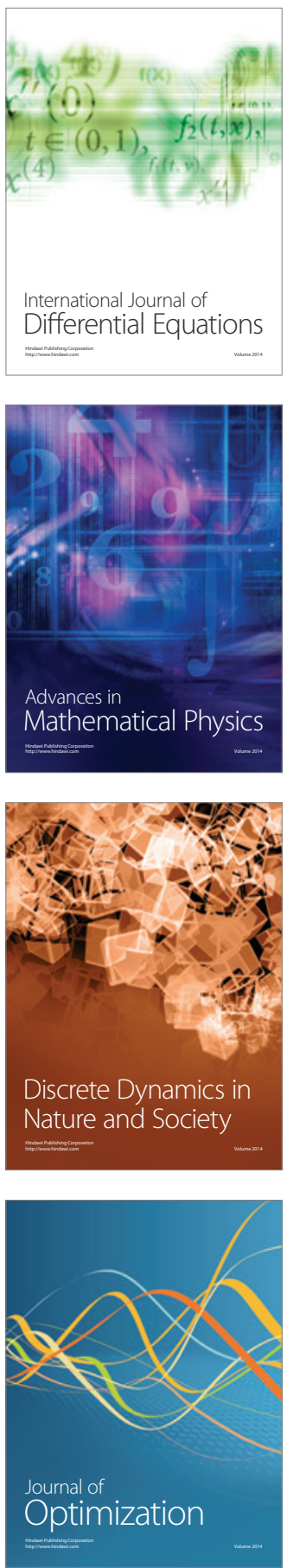